\title{
Application Caching \& Clustering Techniques for Upgrading the Data Retrieval Performance in Mobile Ad Hoc Networks (Manets)
}

\author{
Y. J. Sudha Rani ${ }^{1 *}$, B. Ravi Raju ${ }^{2}$ \\ ${ }^{1}$ Research Scholar, Jntuh, Department of Cse, Smich, Hyderabad, India \\ ${ }^{2}$ Assistant Professor, Department of It, Agi, Hyderabad, India
}

*Corresponding Author: Y. J. Sudha Rani, Research Scholar, Jntuh, Department of Cse, Smich, Hyderabad, India

\begin{abstract}
Mobile advert Hoc Networks (MANETs) provide an appealing answer for networking within the situations wherein community infrastructure or carrier subscription is not to be had. Cooperative caching scheme can improve the accessibility of data gadgets. In this paper, we proposed a Cooperative group data caching scheme called cooperative group data statistics Caching (CGDSC) for improving information access performance in MANETs. The goal is to improve statistics availability and access performance through taking part cooperative sources of cell nodes. Cache resolution and cache management are the two problems of cooperative caching. To improve data availability and access efficiency, cooperative caching discovers statistics resources which set off much less verbal exchange fee by utilising neighbour group nodes. For cache control, cooperative caching increases the powerful ability of cooperative caches by way of minimizing caching duplications in the cooperation region and accommodating extra information types. We examine the performance of the organization facts Caching with the aid of the usage of GCC and compare it with the existing schemes including Neighbor caching and Zone Cooperative. The experimental outcomes show that the cache hit ratio is expanded by about eleven $\%$ 40\% and the query delay about $34 \%$ finally the average latency is reduced with the aid of about five\% 37\% compared with other schemes.
\end{abstract}

Keywords: caching duplication, AdHoc, Cache management, cooperative caching

\section{INTRODUCTION}

A mobile ad hoc network (MANET), also known as wireless ad hoc network or ad hoc wireless network, is a consistently self-arranging, foundation less system of cell phones associated remotely. Every gadget in a MANET is allowed to move freely toward any path, and will in this manner change its connects to different gadgets as often as possible. Each must forward activity irrelevant to its own utilization, and in this way be a switch. The essential test in building a MANET is preparing every gadget to consistently keep up the data required to appropriately course traffic.[4] Such systems may work independent from anyone else or might be associated with the bigger Web. They may contain one or numerous and diverse handsets between hubs. This outcomes in an exceptionally powerful, self-sufficient topology. [4]

MANETs are a sort of remote specially appointed system (WANET) that for the most part has a routable systems administration condition over a Connection Layer impromptu system. MANETs comprise of a distributed, self-framing, self-recuperating system. MANETs around 2000- 2015 normally impart at radio frequencies $(30 \mathrm{MHz}-5 \mathrm{GHz})$.

MANET is a collection of wireless adaptable nodes basic a acting network without the charge for abject stations or any added preexisting network infrastructure. In a peer-to-peer fashion, mobile nodes can acquaint with anniversary added by application wireless multi hop communication. Due to its low cost, high flexibility, fast arrangement enactment and self re configuration, ad hoc networking has accustomed much interest during the endure ten years. However, after a fixed infrastructure, common aisle changes could cause significant numbers of acquisition packets to ascertain new paths, leading to added manual cessation over anchored networks. 

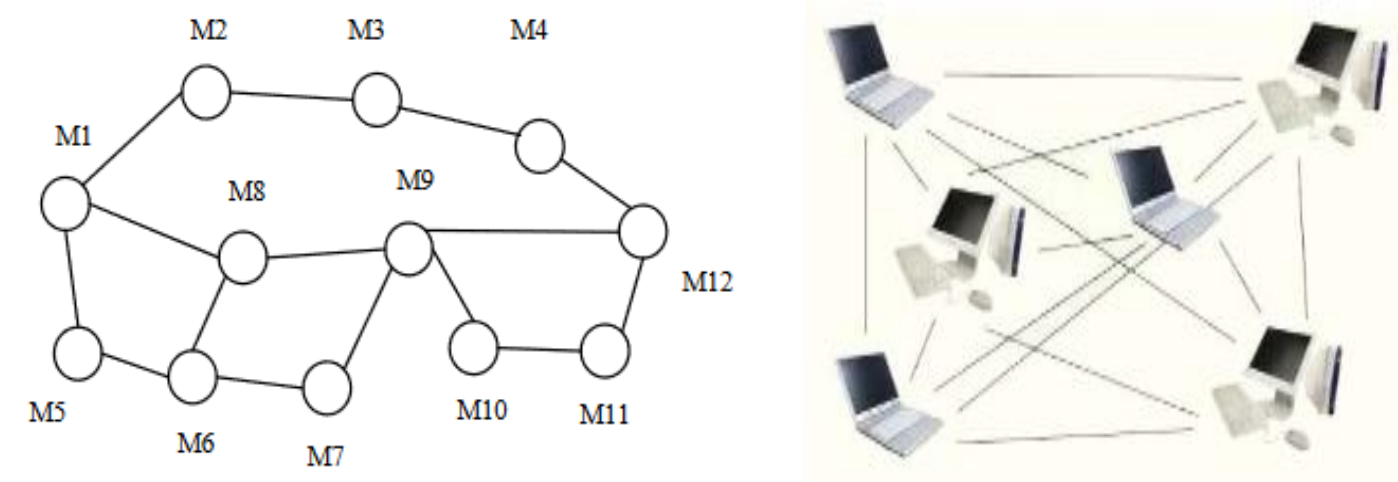

Figure1. Mobile Ad-hoc network

Each MANET bulge can serve as a router, and may move arbitrary and dynamically affiliated to anatomy network depending on their positions and manual range. The topology of the ad hoc arrangement depends on the transmission ability of the nodes and the area of the MNs, which may change with time. Caching techniques are an able band-aid for accretion the achievement in message or abstracts communication [3]. The aboriginal abstraction of caching is that the abstract accessed by MHs has the properties of banausic and spatial locality. Higher temporal and spatial belt ensures that a lot of accesses will go to the abstracts that were accessed afresh in the past and that abide in the cache. Therefore, caching frequently requested abstracts can advance the achievement of data communication.

\section{RELAVENT WORKS ON MANETS}

\subsection{Cooperative Caching}

A number of data replication schemes and caching schemes have been proposed in order to facilitate data access in mobile Ad hoc networks (MANETs). Data replication studies the issue of allocating replicas of data items to meet access demands. These techniques normally require a priori knowledge of the network topology. Caching schemes however do not facilitate data access based on the knowledge of distributed data items. In Simple Cache the requested data item has always been cached by the requester node. The node uses the cached copy in order to serve subsequent requests when they arrive. The requester node has to get the data from the data center in case of cache miss [6]. However increasing the hop distance between the requester node and caching node will increase the response time for the request. In the research area of mobile Ad hoc networks have been developed a number of caching protocol.

\subsection{Coach Path and Coach Data}

Coach path and data are the intermediate hosts, which are located on the trail between the supply host and also the destination host, cache often accessed knowledge things. In Cache Data, the router node caches the information rather than the path once it finds that the information is usually accessed. Cache Data enforces another rule: A node doesn't cache the data if all requests for the information square measure from a similar node. The Cache Data approach desires additional area to save lots of the data, it ought to be used providentially. In Cache Path, the intermediate hosts record the routing path info of passing knowledge. Cache Path solely records the information path once it is nearer to the caching host than the information supply. To handle cache consistency, Cache Path and Cache Data use a simple weak consistency model supported the time-to-live mechanism. During this model, a routing node considers a cached copy up-to-date if its TTL hasn't invalid. If the TTL has invalid, the node removes the map from its routing table (or removes the cached data). As a result, the routing node forwards future requests for this knowledge to the data supply. We tend to optimize this model by permitting nodes to refresh a cached knowledge item if a recent copy of a similar knowledge passes by. If the recent copy contains a similar knowledge however a newer TTL, the node updates solely the cached data's TTL field. If the information center has updated the information item, the node replaces each the cached knowledge item and its TTL with the fresh copy. 


\subsection{Zone Cooperative}

The Zone Cooperative [8] scheme considers the progress of data discovery. In ZC, each client has a cache to store the frequently accessed data items. The data items in the cache satisfy not only the client's own requests but also the data requests passing through it from other clients. For a data miss in the local cache, the client first searches the data in its zone before forwarding the request to the next client that lies on a path towards server. Zone cooperative (ZC) caching scheme for data retrieval in mobile ad hoc networks. The ZC caching uses a simple weak consistency model based on the time-tolive (TTL), in which a client considers a cached copy up-to-date if its TTL has not expired. The client removes the cached data when the TTL expires. A client refreshes a cached data item and its TTL if a fresh copy of the same data passes by. However, the latency may become longer if the neighbors of intermediate nodes do not have a copy of the requested data object for the request.

\subsection{Group Caching}

In Group Caching (GC), each node and its one - hop neighbors form a cluster [7] among transmission vary by causing the "Hello" messages sporadically. Every cluster features a master node which has to communicate with its members directly through one -hop routing. It increases the communication speed and reduces the delay among nodes in the cluster. In order to utilize the cache house of every node in an exceedingly cluster, the master node checks the caching standing of cluster members mistreatment caching management message. Hence, it stores a lot of different information things and increases the information accessibility. In order to record the caching status of cluster members, every node maintains self table, group table to record caching standing of itself and its cluster members respectively. Once a $\mathrm{MN}$ receives the request, it checks the self table for native cache hit. When native cache miss, checks the group table to send the request to cluster members for remote cache hit. When remote cache hit, the request send to the next group on the path to the server. Once lay to rest mediate cluster along the trail receives the request, it checks its cluster table for Middleware Application Layer Cache Layer (Cache Resolution, Cache Management).In this paper, the proposed group-based cooperative caching scheme (GCC) is based on the concept of group caching as in the many existing caching algorithms stated earlier.

\section{COMParison OF CoOperative CACHING SCHEMES}

Cache Hit Ratio, Average Query Delay and Mean Query Generate Time are some of the performance metrics used to evaluate the performance of the data access.

Cache Hit Ratio: The percentage of accesses that result in cache hits is known as the hit rate or hit ratio of the cache.

Average Query Delay: The time interval between the time of generating a query in the requester and the time of receiving requested data object from the data source.

Table provides a comparison of Cooperative Caching Schemes for data retrieval in MANETs. The Hybrid Cache and Group Caching deal with Cache Consistency. In order to have efficient information retrieval architecture, it is better to incorporate strong consistency in the caching schemes.

Table1. Comparison of various cooperative Caching systems

\begin{tabular}{|l|l|l|}
\hline Cooperative Caching Strategy & \multicolumn{1}{|c|}{ Performance Metrics } & \multicolumn{1}{c|}{ Cache Management } \\
\hline $\begin{array}{l}\text { Cache Data, Cache Path, } \\
\text { Hybrid Cache }\end{array}$ & $\begin{array}{l}\text { Reduces the query delay and the } \\
\text { message complexity. }\end{array}$ & $\begin{array}{l}\text { Cache Replacement and Cache } \\
\text { Consistency }\end{array}$ \\
\hline COOP & $\begin{array}{l}\text { Improves data request success } \\
\text { ratio and average response delay. }\end{array}$ & $\begin{array}{l}\text { Cache Admission Control and } \\
\text { Cache Discovery }\end{array}$ \\
\hline CC Caching & $\begin{array}{l}\text { Reduces the message overheads } \\
\text { and enhances the data } \\
\text { accessibility. }\end{array}$ & $\begin{array}{l}\text { Cache Admission Control and } \\
\text { Cache Discovery }\end{array}$ \\
\hline Group Caching & $\begin{array}{l}\text { Increases the hit ratio and } \\
\text { reduces the access latency Cache Discovery, Cache Placement, } \\
\text { Cache } \\
\text { Cache replacement, } \\
\text { consistency }\end{array}$ \\
\hline COACS & $\begin{array}{l}\text { Improves cache hit ratio and } \\
\text { average query latency }\end{array}$ & $\begin{array}{l}\text { Cache Discovery, Cache Admission } \\
\text { Control, Cache Replacement }\end{array}$ \\
\hline
\end{tabular}


Application Caching \& Clustering Techniques for Upgrading the Data Retrieval Performance in Mobile Ad Hoc Networks (Manets)

Table2. Hit ratio and Query delay of caches

\begin{tabular}{|c|c|c|}
\hline Cache Scheme & Hit Ratio (\%) & Query Delay (Sec.) \\
\hline Cache Data & 0.7 & 0.13 \\
\hline Cache Path & 0.7 & 0.18 \\
\hline Hybrid Cache & 0.8 & 0.11 \\
\hline Group Caching & $11 \%$ & 34 \\
\hline ZC Caching & 0.8 & 0.14 \\
\hline COACS & 0.7 & 0.7 \\
\hline
\end{tabular}

\section{Clustering in Manets}

A fruitful strategy for managing the upkeep of MANETs is by dividing the system into groups. Along these lines the system turns out to be more sensible. Bunching is a technique which totals hubs into bunches known as Groups (B.An et al., 2001). A group is fundamentally a subset of hubs of the system that fulfills a specific property and closely resembling cells in a cell arrange. Be that as it may, the group association of an impromptu system couldn't be accomplished disconnected as in settled systems (A. McDonald et al., 1999; L. Wang et al., 2005) Bunching presents a few focal points for the medium access layer and the system layer in MANET (T. C. Hou et al., 2001). The usage of bunching plans permits a superior execution of the conventions for the Medium Access Control (MAC) layer by enhancing the spatial reuse, throughput, versatility and power utilization, grouping enhances directing at the system layer by diminishing the extent of the steering tables and by diminishing transmission overhead because of the refresh of directing tables after topological changes happen (L.Wang et al., 2005). In this manner, every hub stores a small amount of the aggregate system steering data. The motivation behind a grouping calculation is to deliver and keep up an associated bunch (G. Lugano, 2003). In most grouping systems, hubs are chosen to assume distinctive parts as per a specific criteria.

\section{Clustering Schemes FOR MANETS}

The vast majority of the bunching plans depend on the diagrams. A couple of plans utilize fluffy set hypothesis to frame dynamic groups. In this segment the audit of the bunching plans for the MANET is exhibited.

A hub with the base id is picked as a bunch head in the Lowest ID group calculation (LIC) in which the neighbors of the bunch head have the higher ID than that of the group head (A. Ephremides et al., 1987). A hub which exists in the transmission scope of at least two bunch heads will go about as an entryway hub which is utilized for directing between groups. Every hub is relegated with a particular id.

The Lowest-ID conspire worries with the most minimal hub ids which are subjectively doled out numbers without considering some other capabilities of a hub for decision as a group head. Since the hub ids don't change with time. In this manner, downside of least ID calculation is that sure hubs are inclined to control seepage because of filling in as bunch sets out toward longer timeframes.

The most elevated degree heuristic depends on the availability between a hub and its immediate neighbors. Every hub communicates occasionally its availability incentive to its neighbors. A hub chooses to wind up a group head or remain a conventional hub by contrasting the availability estimation of its neighbors with its own esteem. On the off chance that a hub has the most astounding availability esteem in its neighborhood it will end up being a bunch head (M. Gerla et al., 1995).

G. Chen et al. (2002) proposed the consolidated calculation of the Lowest-ID and the Highest-degree heuristics as K-CONID. The availability and the lower ID are considered as the measure for the choice of bunch heads. The use of hub availability as a model causes various ties between hubs. Then again, utilizing just a lower ID foundation creates a bigger number of bunches than would normally be appropriate. This calculation limits the quantity of bunches framed in the system and along these lines acquires overwhelming arrangements of littler sizes. Bunches in the k-CONID approach are framed by a group head and all hubs that are at remove at most k-bounces from the group head.

\section{Performance Evaluation Metrics}

The most critical issue while assessing the execution of any convention is identified with the measurements that are utilized for the assessment. In this work, we construct our assessment criteria 
in light of the IETF proposals in RFC 2501 [12]. As proposed, directing conventions for MANETs ought to be assessed on both subjective and quantitative measurements. Subjective measurements portray attractive conventions' properties that make them productive for use in the impromptu remote condition. Quantitative measurements incorporate factual information, which give the devices to evaluate the execution of the directing conventions. The information ought to be connected with the progression of the impromptu remote system. We will begin our assessment procedure by looking at the most understood steering conventions by utilizing subjective measurements. At that point, the chose conventions will be tried through reproductions with the ns- 2 .

\subsection{The Simulation Model}

The simulation is performed on NS2 with the CMU wireless extension. In our simulation, the AODV routing protocol [7] was tested as the underlying ad hoc routing algorithm. The simulation time is set 6000 seconds. The number of mobile hosts is set to 100 in a fixed area. It is assume that the wireless bandwidth is $2 \mathrm{MB} / \mathrm{s}$ and the radio range is $100 \mathrm{~m}$. There are totally 1000 data items distributed uniformly among all MHs. The number of hot data objects is set to 200 and all hot data objects are distributed uniformly among all MHs. The probability of queries for the hot data is set to $80 \%$. The query rate of MHs is set to $0.2 /$ second. In order to simulate the node join and leave operations, we set a join/leave rate. If the value of join/leave rate is 20 , there will be ten MHs randomly joining and leaving the network every 20 seconds. If an MH joins or leaves the network, its content of cache will be cleared. We model the movement of nodes in a $1500 \mathrm{~m}$ x $500 \mathrm{~m}$ rectangle area. Mrs. K. Shanmugavadivu et al highlighted the simulation models of the effect of delivery ratio on different node leave and join rate. They are also highlighted about the effect of average query latency on number of nodes and effect of average query latency on different node leave/Join rate.

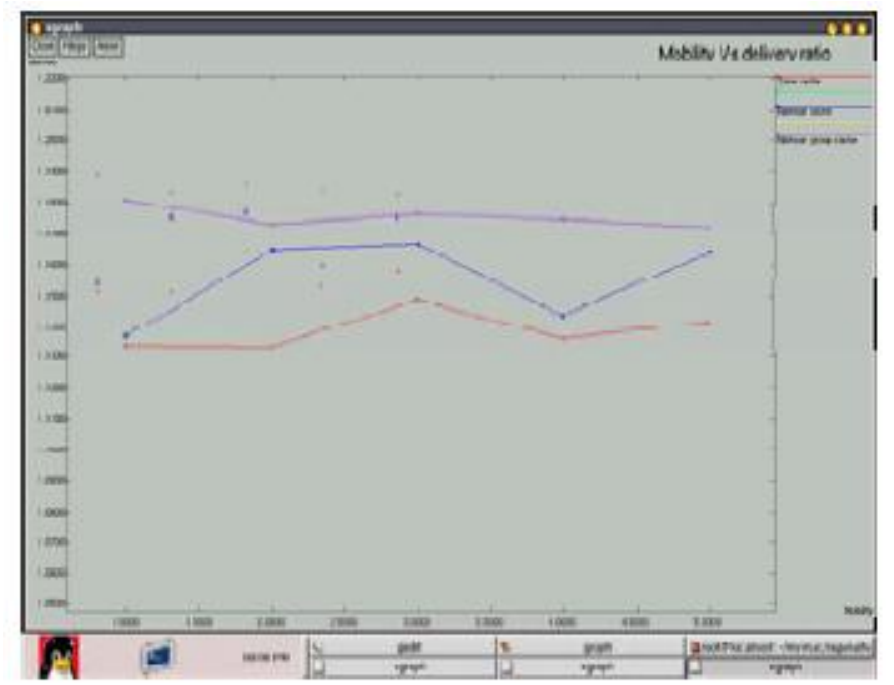

Figure2. Effect of delivery ratio on different node leave/Join rate [10]

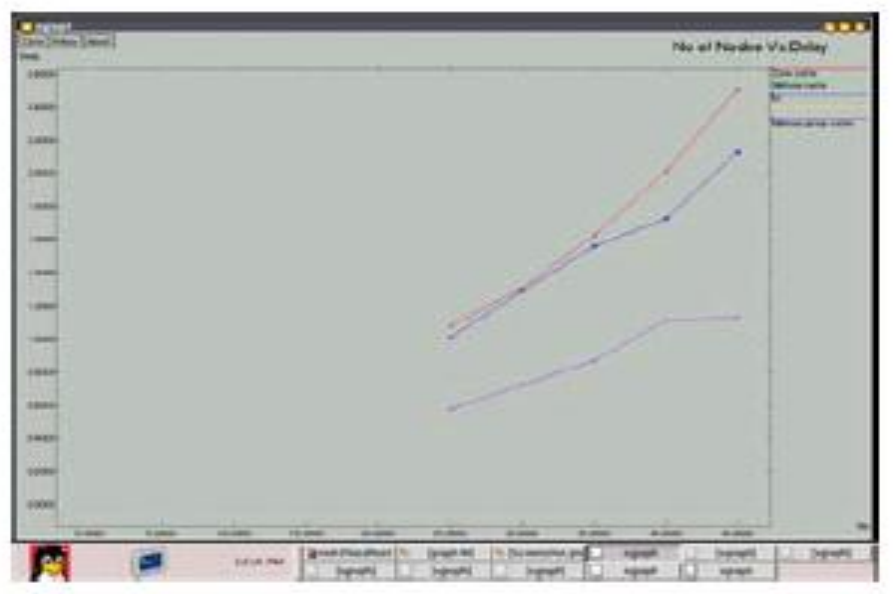

Figure3. Effect of average query latency on number of nodes [10] 


\section{CONCLUSION}

- An analysis and comparison on the cooperative caching schemes and clustering Schemes and performance evaluation in MANET are presented in this paper.

- Cooperative caching provides sharing of data among the nodes and improves data accessibility.

- Clustering mechanisms reduces routing overhead. The integration of cooperative caching schemes and clustering mechanisms provides more efficiency and data accessibility. Data replication takes place in the caching nodes when caching is used.

- The caching schemes that are discussed are not efficient during the time of change in topology and weak in security. As the mobile nodes is responsible for returning the cached data, or modify the route and forward a request to the caching node, it is very important to monitor the malicious nodes and node failure. The data transmission should be fast and secure.

- Thus caching plays a vital role in the efficient communications in MANET. It is ascertained that various aspects of caching in MANET and the advantages of caching and cache invalidation mechanisms that optimize data availability in MANET besides improving throughput and decreasing computational overhead.

\section{REFERENCES}

[1] C. Siva Ram Murthy and B.S. Manoj" Ad-hoc wireless network architectures and protocols".

[2] Cao, G., L. Yin, and C. Das. "Cooperative Cache-Based Data Access in Ad Hoc Networks." IEEE Computer, 2004.

[3] Y. Hu, D.B. Johnson, "Caching Strategies in On Demand Routing Protocols for Wireless Ad Hoc Networks," in Proceedings of the 6th Annual International Conference on Mobile Computing and Networking (MobiCom), Aug.

[4] Kuppusamy, P. and B. Kalaavathi. (2012). Cluster Based Data Consistency for Cooperative Caching over Partitionable Mobile Adhoc Network. ISSN. 9 (8), p1307-1315.

[5] Yu Huang, Beihong Jin, Jiannong Cao, Guangzhong Sun, Yulin Feng. (n.d). A Selective Push Algorithm for Cooperative Cache Consistency Maintenance over MANETs. IEEE. p1-11.

[6] F.J. Gonzalez-Cañete and E. Casilari. (2011). Impact of the Mobility Model on Cooperative Caching Scheme for Mobile Ad Hoc Networks. inTech. p266- 285.

[7] Roberto Beraldi and Roberto Baldoni. (2003). A Caching Scheme for Routing in Mobile Ad Hoc Networks and Its Application to ZRP. Published by the IEEE Computer Society. 52 (8), p1-12.

[8] . Hara, T. (2002). Replica allocation in ad hoc networks with periodic data update. In Proceedings of the 3rd International Conference on Mobile Data Management (pp. 79-86).

[9] Lim, S., Lee, W. C., Cao, G., \& Das, C.R. (2003). A novel caching scheme for internet based mobile ad hoc networks. In Proceedings of the IEEE (ICCCN), (pp. 38-43).

[10] Mrs. K.Shanmugavadivu 1 and Dr M.Madheswaran2, Caching Technique for Improving Data Retrieval Performance in Mobile Ad Hoc Networks, International Journal of Computer Science and Information Technologies, Vol. 1 (4) , 2010, 249-255

Citation: Y. J. Sudha Rani, B. Ravi Raju. (2018) "Application Caching \& Clustering Techniques for Upgrading the Data Retrieval Performance in Mobile Ad Hoc Networks (Manets)", International Journal of Innovative Research in Electronics and Communications (IJIREC), 5(2), pp 49-54. DOI: http://dx.doi. org/10.20431/2349-4050.0502004

Copyright: () 2018 Y. J. Sudha Rani, B. Ravi Raju, This is an open-access article distributed under the terms of the Creative Commons Attribution License, which permits unrestricted use, distribution, and reproduction in any medium, provided the original author and source are credited. 